\title{
Relation of roots and trunks of brachial plexus to scalenus anterior muscle and its clinical significance
}

\author{
Yogesh $^{1}$, Viveka $\mathrm{S}^{2}$, Sudha M J ${ }^{3}$, Santosh Kumar S.C ${ }^{4}$, Sanjay Revankar ${ }^{5}$ \\ ${ }^{1}$ Assistant Professor, Department of Anatomy, Shridevi Institute of Medical Sciences \& Research Hospital, Tumkur \\ ${ }^{2}$ Assistant Professor, Department of Anatomy, Azeezia Institute of Medical Sciences, Kollam \\ ${ }^{3}$ Assistant Professor, Department of Pharmacology, Azeezia Institute of Medical Sciences, Kollam \\ ${ }^{4}$ Department of Pharmacology, Shridevi Institute of Medical Sciences \& Research Hospital, Tumkur \\ ${ }^{5}$ Post graduate, Department of Anatomy, A J Institute of Medical Sciences, Mangalore.
}

\begin{abstract}
Variations in the structures at the root of neck are important in understanding many clinical and surgical conditions. Scalenus anterior, the key muscle in the neck, usually related to the roots of brachial plexus in its posterior aspect. This study was designed to evaluate the relations of roots of brachial plexus to the scalene muscles. Posterior triangles of neck on both sides were studied in 24 cadavers. In two cases C5 and C6 pierced scalenus anterior muscle and emerged from its anterior surface. In other specimen roots of C5, C6 and $C 7$ entered scalenus muscle and exited anterolateraly in a sequential manner. Knowledge of such variations is important for anaesthetists and surgeons.
\end{abstract}

Key words: Scalenus anterior; Scalenus medius; roots of brachial plexus; variations

\section{Introduction}

Brachial plexus is formed by union of ventral rami of lower four cervical nerves and first thoracic nerve. After exit out of intervertebral foramen, these ventral rami usually run in the interval between scalenus anterior and scalenus medius. The fifth and sixth rami unite at the lateral border of scalenus medius as the upper trunk; the eighth cervical and first thoracic rami join behind scalenus anterior as the lower trunk; the seventh cervical ramus becomes the middle trunk. Scalenus anterior muscle is attached to anterior tubercles of transverse processes of third, fourth, fifth and sixth cervical vertebrae through musculotendinous fasciae. Its lower end is attached to scalene tubercle on inner border of clavicle. This muscle forms an important structure in the neck, having phrenic nerve anterior to it and roots of brachial plexus and subclavian artery posterior to it. Another 'ladder' muscle of the neck, located posterior to this muscle, scalenus medius is attached to transverse process of the axis and the front of the posterior tubercles of the transverse processes of the lower five cervical vertebrae. Lower end of medius muscle is attached to upper surface of the first rib between the tubercle of the rib and the groove for the subclavian artery. Scalenus medius being the largest and longest of scalene muscles is related anteriorly to roots of brachial plexus and subclavian artery.

After leaving the intervertebral foramina, the anterior primary rami of the nerves destined to become the brachial plexus travel in the gutter formed by the anterior and posterior tubercles of the corresponding transverse processes of the cervical vertebrae. After leaving the transverse process, the roots of the plexus descend in front of the middle scalene muscle. The prevertebral fascia splits and invests around these two scalene muscles, enclosing a space between the, referred as scalene hiatus. This fascia also ensheaths the intervening roots of ventral rami of nerves destined to become the brachial plexus. There are many attempts in the past to study the variations of brachial plexus and relation of plexus with first rib, carotid and subclavian arteries. Brachial plexus has been spatially mapped using MR imaging technique [1].

The interscalene block entails anesthetizing the brachial plexus at the level of the nerve roots of C5-6 or superior trunk as it lies between the anterior and medial scalene muscles in the neck. This technique was developed by Dr. AlonWinnie [2]. Interscalene brachial plexus block is indicated for surgical procedures of shoulder joint and upper arm. This technique is also gaining importance in shoulder arthroscopy. Injection of local anesthetic into the plane between scalenus anterior and scalenus medius results in spread within this layer both rostrally towards $\mathrm{C} 2$ and caudally toward $\mathrm{T} 1$. Interscalene block is typically performed at the midpoint C56 so that spread upward involves the upper cervical plexus (C2-4) and downward affects the brachial plexus (C5-T1). There are very few studies which evaluates relation of roots and trunks of brachial plexus with scalene muscles. Knowledge of relation of roots with scalene muscles is clinically significant as these muscles may form scissor like compression upon contraction. Passage of roots and trunks within any of the scalene muscles may result in chronic pain syndromes of neck. This study was designed to appreciate relationship of roots and trunks of brachial plexus with scalene muscles in cadavers. 


\section{Methodology:}

The posterior triangles of neck on both sides were carefully dissected in 24 cadavers and head and neck specimens from department of Anatomy, Azeezia Institute of Medical Sciences, Kollam. After removal of skin, platysma muscle and investing layer of deep cervical fascia over roof of posterior triangle, its contents were noted and roots and trunks of brachial plexus were traced proximally to the scalene muscles. Its relation to the scalene muscles noted.

\section{Results:}

Out of 48 posterior triangles examined, roots of brachial plexus were present in the scalene hiatus, i.e. in the interval between scalene anterior and scalene medius in 46 sides. In 3 instances, the roots were present in the substance of scalene anterior muscle. In all the cases the roots were traced proximally towards intervertebral foramen, it was noted that ventral rami of $\mathrm{C} 5,6$ and 7 were entering the scalenus anterior muscle from its posterior surface in one of the specimen. The upper two ventral rami emerged from the anterior surface and lower ones sequentially shifted towards lateral border of the scalenus anterior muscle. In other 2 specimens C5 and C6 were emerging from the anterior surface of scalenus anterior muscle. The fascia over the roots blended with prevertebral fascia of posterior surface of scalenus anterior.

\section{Discussion:}

Cadaveric studies of brachial plexus reported in the literature mainly concentrates on number, position, relations of cords and branches and formation of brachial plexus. The knowledge about relations of roots with respect to scalene muscles is important for surgeons and anaesthetists. Inter individual variations of the roots in the interscalene space may lead to differential grades of anaesthesia after supraclavicular brachial plexus block.

The present study shows that the roots can be embedded in the substance of the scalene muscle instead of locating in the interval between the muscles. In a similar cadaveric study by Walter G. Harry and et al, reports that that roots of $\mathrm{C} 5$ and $\mathrm{C} 6$ unite before entering scalenus muscle in most of cases $(15 \%)$ where they are not present in the interscalene interval $(60 \%)$. There are many studies by anaesthetists examining the location of roots of brachial plexus as it forms the prime importance in supraclavicular brachial plexus block using either ultranography[3,4,5] or CT scans [6]. But these studies seldom concentrate on the relation of roots to scalene muscles. Ting in his study of spread of local anesthetic in axilla after supraclavicular block has concentrated more on inferior spread of drug, which is of more functional significance than on superior spread [7]. Study of proximal spread of the drug would have shown light on relation of roots of brachial plexus with scalene muscles. Roots of brachial plexus going through the muscle substance may undergo scissor like compression from muscular contraction and in turn lead to chronic pain often radiating in the area their supply.

\section{Conclusions:}

This study clearly demonstrates that variations in the course of roots of brachial plexus do exists in relation to the scalene muscles and such variations should be given due importance by anaesthetists, surgeons and radiologists working in the roots of neck.

\section{References:}

[1] Posniak HV, Olson MC, Dudiak CM, Wisniewski R and C O'Malley. MR imaging of the brachial plexus. American Journal of Roentgenology. 1993;161: 373-379.

[2] Winnie AP. Interscalene brachial plexus block. Anesth Analg. 1970; 49:455-466.

[3] Perlas A, Chan VW, Simons M. Brachial plexus examination and localization using ultrasound and electrical stimulation: a volunteer study. Anesthesiology. 2003; 99:429-35.

[4] De Andres J, Sala-Blanch X. Ultrasound in the practice of brachial plexus anesthesia. Reg Anesth Pain Med. 2002; 27:77-89.

[5] Chan VW. Applying ultrasound imaging to interscalene brachial plexus block. Reg Anesth Pain Med. 2003; 28: 340-343.

[6] Mukherji SK, Wagle A, Armao DM, Dogra S. Brachial plexus nerve block with CT guidance for regional pain management: initial results. Radiology. $2000 \mathrm{Sep} ; 216(3): 886-90$.

[7] Ting PL, Sivagnanaratnam V. Ultrasonographic study of the spread of local anaesthetic during axillary brachial plexus block. $\mathrm{Br} J$ Anaesth. 1989 Sep;63(3):326-9. 


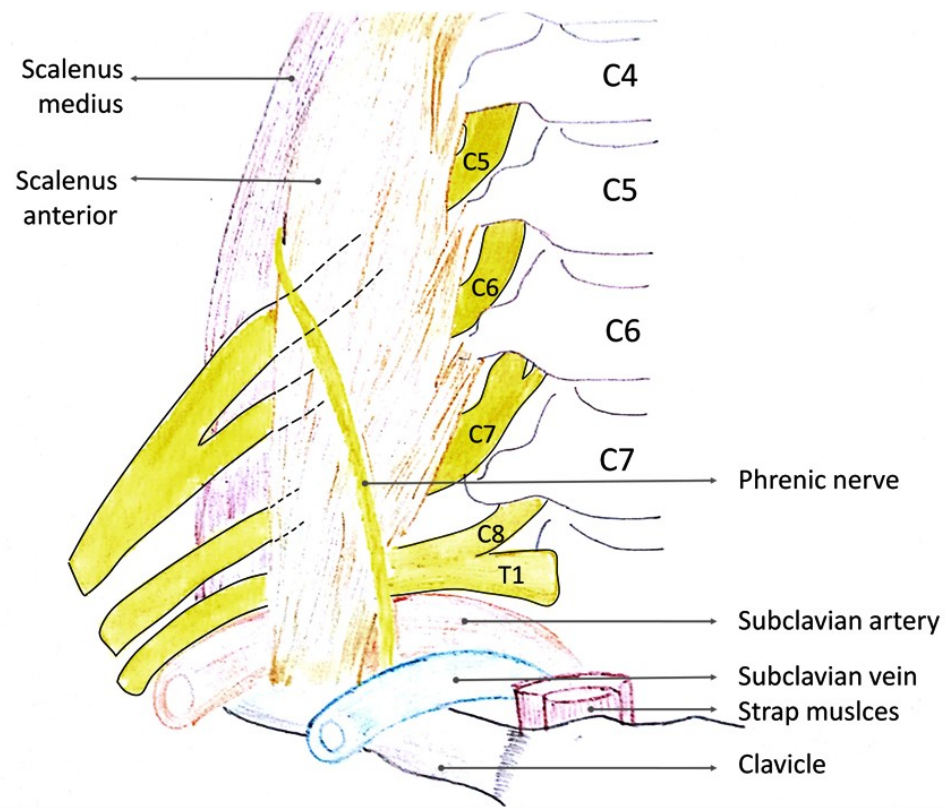

Figure 1: Schematic diagram showing variations in relations of roots of brachial plexus to scalenus anterior muscle. Variations are depicted in dashed lines. 